\title{
Biological Paternal Grandfather
}

National Cancer Institute

\section{Source}

National Cancer Institute. Biological Paternal Grandfather. NCI Thesaurus. Code C96583.

A male relative who is the biological father of the biological father. 